\title{
Pyrazinamide Induced Parkinsonism
}

\author{
FARZANA SHUMY, ${ }^{1}$ AHMAD MURSEL ANAM, ${ }^{2}$ M A JALIL CHOWDHURY, ${ }^{3}$ MD. NAHIDUZZAMANE SHAZZAD ${ }^{1}$
}

\begin{abstract}
:
"Parkinsonism" refers to a syndrome characterized by the presence of tremor, rigidity and bradykinesia. In addition there is loss of postural reflexes as well as freezing. Drugs rank the second in the causes of parkinsonism. As it is reversible, drug-induced parkinsonism (Rabbit syndrome) should be borne in mind and suspected in any patient receiving drug treatment. We report a case of drug induced parkinsonism, evidently due to pyrazinamide. To the best of our knowledge, this is the first case to be reported in our country.
\end{abstract}

Keywords: Pyrazinamide, Drug induced parkinsonism, Rabbit syndrome.

\section{Introduction}

Since the description of a shaking palsy by James Parkinson in 1817 , different explanations have evolved, until the term "parkinsonism" referred to a syndrome characterized by the presence of tremor, rigidity, and bradykinesia in addition to loss of postural reflexes and freezing. ${ }^{1}$ Drugs rank the second in causes of parkinsonism. ${ }^{2}$ As it is reversible, drug-induced parkinsonism should be borne in mind and suspected in any patient receiving drug treatment. ${ }^{1}$

\section{Case report}

In mid-December, 2013, a 52-year-old man, from a remote village of Bangladesh, presented to the Bangabandhu Sheikh Mujib Medical University (BSMMU) hospital, Dhaka, for evaluation of fever for six months. Before BSMMU, he had thorough medical evaluation in different local and regional hospitals, but no conclusion could be drawn on the cause of his fever. CAT-1 anti-tubercular chemotherapy was commenced empirically in another hospital by the end of November, 2013. But the patient had to discontinue it after about a week due to severe nausea. After being admitted in BSMMU, despite all possible efforts, again, no definitive cause could be determined for his fever. Empirical CAT-1 anti-tubercular chemotherapy was re-started. That time in

1. Medical Officer, Department of Internal Medicine, Bangabandhu Sheikh Mujib Medical University (BSMMU), Shahbagh, Dhaka.

2. Chief Resident, Intensive Care Unit, Square Hospitals Ltd. 18/F, BU Qazi Nuruzzaman Sarak, Dhaka 1205, Bangladesh.

3. Professor \& Chairman, Department of Internal Medicine, BSMMU, Dhaka.

Corresponding Author: Dr. Farzana Shumy, MBBS, FCPS (Medicine). Medical Officer, Department of Internal Medicine, Bangabandhu Sheikh Mujib Medical University, Shahbagh, Dhaka 1000, Bangladesh. Mobile: 01911010841, e-mail: farzanashumy @ hotmail.com
WHO recommended challenging dose, to single out the drug that caused nausea and lead to the discontinuation of this regimen earlier. On the second day of administration of pyrazinamide (in challenging dose: $250 \mathrm{mg}$ on day 1 , and $1000 \mathrm{mg}$ on day 2), he developed bradykinesia, cogwheel and lead-pipe rigidity, tremor (more pronounced in action and posture) affecting right hand, and oro-masticatory dyskinesia. Pyrazinamide was discontinued immediately, and Tab. Procyclidine (5 mg 8 hourly) was commenced. All the extrapyramidal symptoms resolved within five days after withdrawal of Pyrazinamide. Four-drug anti-tubercular chemotherapy, with ofloxacin replacing pyrazinamide was continued, and he was discharged with improving clinical condition. He was found clinically well during his followup two months later, and he reported no further complications in the meantime. Unfortunately, the patient passed away due to a different and unrelated medical condition, after another three months (May, 2014).

\section{Discussion:}

Drug-induced-parkinsonism (DIP) is not only a frequent aetiology of secondary parkinsonism, but also the commonest of all drug-induced movement disorders. ${ }^{2-4}$ Differentiating DIP from Parkinson's Disease (PD) may be difficult in many situations, but certain features help to establish the correct diagnosis. ${ }^{2} \mathrm{DIP}$ is generally characterized by acute/sub-acute onset, symmetric bilateral (sometimes unilateral) involvement, female predominance, more prominent bradykinesia and rigidity, and typical oro-masticatory dyskinesia (Rabbit syndrome - Strongly associated with DIP) ${ }^{2-4}$ DIP has temporal relationship with commencing an offending drug, often within a few days. ${ }^{2}$ Tremor (4-7 $\mathrm{Hz}$ ) in DIP usually affects the upper limbs, and present both at rest and activity, but, is less frequent than PD. ${ }^{3}$ However, clinical manifestations alone cannot distinguish DIP from 
PD. ${ }^{3}$ Imaging of striatal dopamine transporter using SinglePhoton Emission Computed Tomography (SPECT) using radioligand [ $\left.{ }^{123} \mathrm{I}\right] \mathrm{FP}-\mathrm{CIT}$ (showing diminished nigro-striatal dopeminergic cell uptake in PD but not in DIP) may be helpful. ${ }^{3,4}$ But we couldn't do that due to financial constraints. Once DIP has developed, offending drug should be identified and withdrawn. In severe cases, anticholinergic medication may be added. Amantadine is equally effective. ${ }^{2,3}$ Usually, the symptoms resolve within weeks after discontinuation of offenders, but they respond remarkably to anticholinergics. ${ }^{3}$

A long list of offending agents are available. ${ }^{2,3}$ To the best of our knowledge, Pyrazinamide has not been reported as a cause of DIP yet in our country. Different medications cause DIP in different manners, common pathway being reduced dopamine activity, either by dopamine $\left(\mathrm{D}_{2}\right)$ receptor blockade (e.g. Haloperidol, Prochlorperazine, Metoclopramide), depletion of dopamine stores (e.g. Reserpine), dopamine deficiency (e.g. Trimethoprim) or neurotoxicity (e.g. Amiodarone). Nicotinamide deficiency results in dopamine deficiency, loss of cells in the pars compacta, and parkinsonism, with a good response to dopamine agonists. ${ }^{5}$ Pyrazinamide is the most active synthetic analogue of nicotinamide, and the M. tuberculosis nicotinamidase/pirazinamidase (PZAse) enzyme cleaves nicotinamide \& pyrazinamide to their active forms nicotinic acid and pyrazinoic acid, respectively. ${ }^{6}$ Whether this structural similarity and common enzymatic action lead to nicotinamide deficiency, causing pyrazinamide induced parkinsonism, or results from dopamine deficiency by any other possible mechanism is to be determined by further research.

\section{Conclusion}

DIP is frequently overlooked, and patients are prescribed anti-parkinsonian medications unnecessarily. ${ }^{3}$ Proper knowledge as well as high level of suspicion is required for early recognition and proper management of DIP. Physicians, who prescribe anti-tubercular chemotherapy frequently, should remember Pyrazinamide as a possible cause, and act accordingly, if and when faced with a patient of DIP.

\section{Conflict of interest: None.}

\section{References:}

1. Alvarez MV, Evidente VG. Understanding Drug-induced Parkinsonism: Separating pearls from oy-sters. Neurology 2008;70(8):e32-e34.

2. Bohlega SA, Al-Foghom NB. Drug-induced Parkinson's Disease. A Clinical Review. Neurosciences 2013;18(3):215221.

3. Shin HW, Chung SJ. Drug-Induced Parkinsonism. J Clin Neurol 2012;8(1):15-21.

4. Susatia F, Fernandez HH. Drug-Induced Parkinsonism. Curr Treat Options Neurol 2009 May;11(3):162-169.

5. Williams AC, Cartwright LS, Ramsden DB. Parkinson's Disease: the First Common Neurological Disease due to Auto-Intoxication? Q J Med 2005;98(3):215-226.

6. Zhang Y, Mitchison D. The Curious Characteristics of Pyrazinamide: A Review. Int J Tuberc Lung Dis 2003;7(1):621. 